\title{
The Application of Temporary Intestinal Drainage in the Surgical Treatment for Neonates with Necrotizing Enterocolitis
}

\author{
Ji-xue Zhao ${ }^{1, a}$, Xin Fü,b \\ ${ }^{1}$ Department of Pediatric Surgery, The First Hospital of Jilin University, Changchun 130021, China \\ ${ }^{2}$ Nursing Administration Department, China-Japan Union Hospital of Jilin University, Changchun \\ 130033, China \\ aemail: jixuezhao0431@126.com, bemail :xinfu0431@126.com \\ ${ }^{b}$ corresponding author
}

\begin{abstract}
Keywords: Temporary intestinal drainage, Neonates, Necrotizing enterocolitis
Abstract. Object: To explore the feasibility and effects of temporary intestinal drainage in the surgical treatment for the neonates with necrotizing enterocolitis. Methods: A retrospective analysis was conduct on 42 cases of newborns with extensive and multiple intestinal necrosis found intraoperatively, all of which could not accept first-stage resected, treated by traditional operation or by temporary intestinal drainage between January, 2012 to January 2017. Duration of operation, amount of bleeding, and the survival rate after operation were compared between the two groups, which were divided by surgical methods. Results: No significant difference was observed in age, and gender $(\mathrm{P}>0.05)$ between the two groups. However, duration of operation was significantly shorter, the amount of bleeding was significantly lessen, the survival rate was significantly higher in the patients by temporary intestinal drainage than the patients by traditional operation $(\mathrm{P}<0.05)$. Conclusion: To compare traditional operation or temporary intestinal drainage in neonates, a retrospective analysis conducted by us showed the feasibility and effects of temporary intestinal drainage in neonates, and a significant advantage in duration of operation and the survival rate after operation.
\end{abstract}

\section{Introduction}

Necrotizing enterocolitis (NEC) is one of the most common gastrointestinal tract disease frequently affecting premature infant with an incidence of $3 \%$ to $5 \%$, depending on the gestational age (GA) of infant at birth[1,2], and mortality rate of as high as 50\%[3,4]. The main pathological characteristics of NEC is the extensive hemorrhage necrosis of small intestine and the colon. Many NEC cases which have no intestinal necrosis and perforation can get conservative treatment during early stage, and many of them could get successful treatment which has the cure rate of $82.1 \%[5]$, but the data show that $20 \%-60 \%$ of the children still need the surgery[6]. The operation method for the NEC cases is to according to the degree and scope of bowel necrosis.

In most situations, we resect the necrotic bowel and perform the jejunostomy, and 3 months later, ostomy closure will be performed. In the condition that the intestine was affected extensively unable to be resected on the first-stage operation, there are not a definite surgery method which is strongly recommended and agreed. The conventional surgical method is jejunostomy. But surgical time is too long and mortality was relatively high of this method. In view of this kind of situation, we adopted temporary intestinal drainage to replace the conditional jejunostomy in the first stage operation from January of 2012, and achived ideal clinical results.

\section{Patients and methods}

Patients. From January of 2012 to January of 2017, 42 surgically treated neonates with necrotizing enterocolitis in the neonatology department, the First Hospital of Jilin University were enrolled in this retrospective analysis. Each patient signed an informed consent form. Approval was obtained from the institutional review committee of Jilin University. 
Study Design. The patients were divided into two groups according to the surgical methods: 22 patients were treated by traditional method, that is jejunostomy, these patients would accept surgery again 24 hours later to determine whether affected intestine segment could be reserved; 20 patients accepted our novel operation method, that is temporary intestinal drainage, and later an exploratory operation would be conducted to determine whether anastomosis or jejunostomy.

Methods. Traditional methods: Jejunostomy was applied in the first stage in critically affected neonates with NEC, then further operation proceeded 24 hours later to check the severity and length of affected intestine. Necrotic bowel would be resected, and jejunostomy was adopted followed anastomosis after 2 to 3 months. Improved surgery method (temporary intestinal drainage): In the condition of extensive necrotic small intestine and colon that could not be resected in the first stage, we only perform the abdominal washing and temporary intestinal drainage. 7 to 10 days later when the affected newborn's condition was stable, an exploratory surgery could be conducted to determine further surgery method. Primary enteroanastomosis was made when the affected bowel color changed to normal, or Jejunostomy was adopted when affected intestine was unsatisfying followed by enteroanastomosis two or three months later.

Evaluation Criteria of Treatment Effects. Evaluation criteria included the duration of operation and the survival rate after operation.

Statistical Analysis. All measured parameters including the duration of operation and the survival rate after operation, were analyzed by the statistical software program Statistical Product and Service Solutions (SPSS) 17.0 (SPSS Inc., Chicago, IL, USA) and expressed as mean \pm standard deviation $( \pm \mathrm{s})$, and t-test was used. Enumeration data including gender, prevalence frequency were analyzed by $\chi 2$ test. $\mathrm{P}<0.05$ was considered significant.

\section{Results}

Baseline Characteristic. There were no significant difference $(\mathrm{P}>0.05)$ in general data including operation age, gender between the two groups (Table 1.).

Table 1. Baseline characteristics of study patients

\begin{tabular}{cccc}
\hline Characteristic & control group $(15$ cases $)$ & experimental group $(17$ cases $)$ & $P$ value \\
\hline Operation age (days) & $12.9 \pm 2.5$ & $13.3 \pm 3.1$ & non-significant \\
Female & 9 & 8 & non-significant \\
Male & 13 & 12 & non-significant \\
\hline
\end{tabular}

Evaluation of Treatment Effects. The duration of operation of experimental group was obviously shorter than that of control group, and the amount of bleeding of experimental group was obviously less than that of control group $(\mathrm{P}<0.05)$. Among the cases of experimental group, 16 cases got full recovery, and the 4 remainders died, and the survival rate is $80 \%$; Among the cases of the control group, 12 cases got full recovery, and the 10 remainders died, and the survival rate is $54.5 \%$. The survival rate of experimental group was obviously higher than that of control group $(\mathrm{P}<0.05)$. Postoperative follow-up was conducted for 6 months.

\begin{tabular}{lccc}
\hline \multicolumn{4}{c}{ Table 2 Efficacy of the two groups } \\
\hline & $\begin{array}{c}\text { control } \\
\text { group(15cases) }\end{array}$ & experimental group(17 cases) & $P$ value \\
\hline Duration of operation (minutes) & $47.5 \pm 8.6$ & $25.8 \pm 5.5$ & $P<0.05$ \\
Amount of bleeding (ml) & $5.9 \pm 2.6$ & $2.3 \pm 1.6$ & $P<0.05$ \\
survival rate & $12 / 22$ & $16 / 20$ & $P<0.05$ \\
\hline
\end{tabular}




\section{Discussion}

Necrotising enterocolitis (NEC) is an uncommon, but devastating intestinal inflammatory disease that predominantly affects preterm infants. NEC is sometimes dubbed the spectre of neonatal intensive care units, as its onset is insidiously non-specific, and once the disease manifests, the damage inflicted on the baby's intestine is already disastrous. Subsequent sepsis and multi-organ failure entail a mortality of up to $65 \%$ [7]. It is a disorder characterized by ischemic necrosis of the intestinal mucosa, which is associated with inflammation, invasion of enteric gas forming organisms, and dissection of gas into the muscularis and portal venous system [8]. Early stage of NEC mostly could be cured by supportive treatment and antibiotics. Other signs that indicate bowel necrosis must be considered, including unremitting clinical deterioration [9].

However, to critically affected infants who are sensitive to medical treatment, surgical intervention was recommended. And the best operative opportunity is the point when the intestinal wall was necrotized completely without intestinal perforation because this time necrotized bowel could be resected and lessen the intra-abdominal infection. The surgery method of NEC in neonates mainly depends on the severity and length of necrotic intestine [10]. In the condition that the intestine was affected extensively unable to be resected on the first-stage operation, there are not a definite surgery method which is strongly recommended and agreed. The conventional surgical method is jejunostomy. But surgical time is too long and mortality was relatively high of this method. Aim at this situation, we adopted temporary intestinal drainage to replace the conditional jejunostomy in the first stage operation. In the condition of extensive necrotic small intestine and colon that could not be resected in the first stage, we only perform the abdominal washing and temporary intestinal drainage. 7 to 10 days later when the affected newborn's condition was stable, an exploratory surgery could be conducted to determine further surgery method. Primary enteroanastomosis was made when the affected bowel color changed to normal, or Jejunostomy was adopted when affected intestine was unsatisfying followed by enteroanastomosis two or three months later. And this type of surgical method achieved satisfactory clinical effects with a significant higher survival rate of $80 \%$ compared to $54.5 \%$ of the conventional method.

Possible reasons were analyzed and listed as follows. Firstly, the operation time was shorter significantly, and it is a main reason responsible for improved survival rate postoperatively in affected neonates. As we know, neonates' ability to bear long-term surgery and anaesthesia is weaker than older children. Long time operation could directly lead to difficult recovery postoperatively. Then to decrease operation time would be useful, and the improved operation decreased operation time from 47.5 \pm 8.6 minutes to $25.8 \pm 5.5$ minutes. Plus, abdominal washing and temporary intestinal drainage could lesson the tension of bowel wall and reduce the absorption of inflammatory mediators. A reasonable mechanism of NEC is that intestinal mucosa of affected intestine was destroyed, and amounts of inflammatory mediators were generated and absorbed by bowel wall, leading to necrotized intestine, perforation and even multiple organ failure[11]. Therefore, a critical aspect of managing the patients with NEC is to reduce the absorption of inflammatory mediators.

Temporary intestinal drainage could shorten the operation time and apparently reduce the absorption of inflammatory mediators for the serious NEC patients, so this method is feasible and worthy to be popularized in clinic.

\section{Conclusions}

Temporary intestinal drainage, as an easy and feasible method for the serious NEC patients improved by us, has a satisfactory clinical effect with a relatively higher survival rate. And it should be recommended. 


\section{References}

[1] Houben $\mathrm{CH}$, Chan KW, Mou JW, et al. Management of intestinal strictures post conservative treatment of necrotizing enterocolitis: the long term outcome. J Neonatal Surg 2016;5:28.

[2] Heida FH, Loos MH, Stolwijk L, et al. Risk factors associated with postnecrotizing enterocolitis strictures in infants. J Pediatr Surg 2016;51:1126-30.

[3] Moss RL, Kalish LA, Duggan C, et al. Clinical parameters do not adequately predict outcome in necrotizing enterocolitis: a multiinstitutional study. J Perinatol 2008;28:665-74.

[4] Holman RC, Stoll BJ, Curns AT, et al. Necrotising enterocolitis hospitalisations among neonates in the United States. Paediatr Perinat Epidemiol 2006;20:498-506.

[5] Chorna IS, Razumnyi PK, Buryi OM, et a1. Non-invasive methods of control of the intestinal motility in surgical practice[J]. Klin Khir. 1999; (2): 22-25.

[6] Thompson AM, Bizzarro MJ.Necrotizing enterocolitis in newborns: pathogenesis, prevention and management[ J ]. Drugs. 2008;68(9):1227-38.

[7] Al Tawil K. et al. Risk factors, characteristics and outcomes of necrotizing enterocolitis in late preterm and term infants. Journal of Neonatal-Perinatal Medicine, 2013,6, 125-130.

[8] Neu J. Necrotizing enterocolitis: the search for a unifying pathogenic theory leading to prevention. Pediatr Clin North Am 1996; 43:409.

[9] Tepas JJ, Sharma R, Leaphart CL, et al. Timing of surgical intervention in necrotizing enterocolitis can be determined by trajectory of metabolic derangement. J Pediatr Surg 2010; 45:310.

[10]Qinmiao Lin, Yongbiao Wang, ZR Li.Surgical treatment on necrotizing enterocolitis in newborns [J]. Journal of Applied Clinical Pediatrics. 2010, 6 (11) 814-816.

[11] HY Wu, Y Yang. A analysis on risk factors in necrotizing enterocolitis in newborns. Chinese And Foreign Medical Research.2013,11(32):38-39. 